\title{
Observations sur un arrêt rendu en matière de maladies professionnelles
}

\author{
J. HEBERT* \\ (Manuscrit reçu le 22 novembre 1982) \\ RÉSUMÉ
}

Après un rappel de la réglementation française en matière de réparation des maladies professionnelles liées à l'exposition aux rayonnements ionisants et en particulier de la méthode d'estimation de la présomption d'origine, l'auteur fait le point de la jurisprudence antérieure à l'arrêt du 25 février 1982.

Cette jurisprudence fait ressortir que l'intensité de l'action des rayonnements n'est pas une condition de l'exposition habituelle, considérée comme élément primordial de la présomption.

L'originalité de l'arrêt considéré est que pour les dommages non stochastiques l'intensité devient une condition de l'exposition habituelle.

\section{ABSTRACT}

After recalling the French regulations concerning the compensation of occupational diseases linked to ionizing radiation exposure, and particularly about the method of assessment of origin presumption, the author reviews the decision delivered before February 25, 1982. These decisions demonstrate that the radiation effect intensity is not a condition of the regular exposure considered as the most important component of the presumption. The originality of the 1982 judgement is that for nonstochastic damages, the intensity becomes a condition of the regular exposure.

Le 25 février 1982 la Cour d'Appel de Paris a rendu, en matière de maladies professionnelles dues à l'action des rayonnements ionisants (tableau $n^{\circ} 6$ ), un arrêt qui mérite de retenir l'attention (1).

On sait que l'indemnisation en France des maladies professionnelles suppose la réunion de présomptions dont, en particulier, l'exposition habituelle au risque professionnel. Jusqu'à présent, l'exposition habituelle aux rayonnements ionisants devait seulement avoir été d'une certaine régularité et d'une certaine durée. Notre arrêt exige, en outre, une intensité suffisante de l'action de l'agent nocif.

* Président d'honneur de l'Association Internationale de Droit Nucléaire (AIDN).

(1) Inédit, à notre connaissance, et que nous devons à l'amabilité du Dr CI. LEVY. 
Avant de commenter plus en détail l'arrêt précité et d'essayer d'en apprécier la portée, nous supposons que certains lecteurs apprécieront que nous rappelions à grands traits le système de réparation des maladies professionnelles et l'état de la jurisprudence antérieure relative aux maladies professionnelles présumées causées par les rayonnements ionisants.

\section{RAPPEL DES LIGNES DIRECTRICES DU SYSTĖME FRANÇAIS DE RÉPARATION DES MALADIES PROFESSIONNELLES}

"Tout fait quelconque de l'homme, qui cause à autrui un dommage, oblige celui par la faute duquel il est arrivé, à le réparer", dit l'article 1382 du Code civil. Rapproché de la règle de procédure qui oblige le demandeur à apporter les preuves de son bon droit, le système classique de la responsabilité civile apparaît simple, logique, équitable.

Néanmoins, les conditions, notamment sociologiques, dans lesquelles le développement de l'industrie s'est effectué au XIXe siècle, rendaient trop souvent impossible l'administration par les victimes (en particulier les ouvriers) des quatre preuves exigées du demandeur $\left(1^{\circ}\right.$ : existence et quantum du dommage, $2^{\circ}$ : relation causale entre ce dommage et $3^{\circ}$ : un fait -ou une omission ou négligence- du défendeur, $4^{\circ}$ : enfin et surtout que ce fait constitue une faute c'està-dire une déviation par rapport à la conduite à laquelle on aurait pu s'attendre de la part d'un individu raisonnable et prudent placé dans les mêmes conditions objectives).

D'où, à la fin du siècle dernier, l'adoption de systèmes de responsabilité n'exigeant plus la preuve de la faute tant en droit administratif que civil (art. 1384, al. $1^{\text {er) }}$ ou encore en droit social (loi du 8 avril 1898 sur les accidents du travail).

Cette dernière loi renforce la position procédurale des victimes par une présomption. Par ce procédé technique, la loi ou le juge esquive la difficulté de preuve directe d'un fait, par exemple la filiation, en inférant une conséquence juridique: la paternité légitime du mari est déduite d'un autre fait, la conception de l'enfant pendant le mariage, sur la base d'une relation statistique et/ou de considérations politico-morales.

La force des diverses présomptions varie. Dans certains cas, le législateur leur attribue un effet non seulement automatique mais définitif (présomption irréfragable, dans d'autres cas plus nombreux, le défendeur peut démolir a posteriori la présomption en apportant la preuve contraire.

La loi précitée (aujourd'hui Code de la sécurité sociale, art. L 415 et seq.) présume ainsi imputable au travail " l'apparition soudaine au temps et au lieu du travail d'un lésion corporelle" subie par un travailleur.

Depuis 1946, la Sécurité sociale est substituée à l'employeur dans la charge de réparer cet accident. Pour des raisons d'ordre budgétaire cette réparation, quoique meilleure que celle de l'assurance sociale maladie, demeure forfaitaire en règle générale.

Après quarante ans de discussions, une loi du 25 octobre 1919 a adapté le système de présomption de la loi de 1898 à des maladies causées par le plomb ou le mercure dont l'origine est presque toujours d'origine professionnelle.

Mais assez vite (1931 pour les affections provoquées par les rayonnements ionisants), des affections dont l'origine ne se trouve pas toujours dans les condi- 
tions de travail ont été aussi considérées comme maladies professionnelles. Alors que l'on peut assigner une date certaine à la brusque apparition de la lésion réputée accident du travail, la maladie, au contraire, est " le résultat d'une série d'événements à évolution progressive auxquels on ne saurait assigner une origine ou une date précise".

C'est pourquoi, les faits à partir desquels la loi (Code S.S. art. L496 et seq.) présume l'existence d'une maladie professionnelle sont différents de ceux qui constituent la présomption d'imputabilité de l'accident du travail.

Tout d'abord, le régime français de réparation demeure attaché au système dit " de la liste". Alors que toute lésion (une fracture, mais aussi un infarctus, une radiodermite aiguë ou éventuellement le syndrome aigu d'irradiation) peut être présumée accident du travail, une maladie ne sera présumée maladie professionnelle que si elle figure sur la liste du tableau dont se réclame le travailleur, question médicale sur laquelle on nous excusera de glisser.

II faut, de plus, que la première constatation médicale, c'est-à-dire la“ qualification ", le diagnostic de l'affection comme étant une de celle de la liste, intervienne dans un "délai de prise en charge", propre à la maladie considérée et figurant en regard de celle-ci dans une seconde colonne du tableau et qui court à partir de la cessation d'exposition au risque objet du tableau considéré. Ce délai exprime le délai de latence maximal retenu par la réglementation.

Outre ces deux éléments de la présomption qui concernent la qualification et la date d'identification de la maladie, deux autres éléments concernant les conditions de travail sont exigés.

Le travailleur doit avoir effectué, avant le point de départ du délai de prise en charge, un travail susceptible de l'exposer aux maladies énumérées dans le tableau dont il se réclame. Ces travaux sont aussi énumérés dans chaque tableau, mais dans certains tableaux, dont le tableau $n^{\circ} 6$, cette liste n'est qu'indicative et se conclut par la formule très générale " tous travaux exposant à l'action des rayons $\mathrm{X}$ ou des substances radioactives, naturelles ou artificielles ou à toute autre source d'émission corpusculaire".

Mais ce laxisme dans l'énumération des travaux est contrebalancé par la dernière condition. II faut que l'exposition à l'agent nocif ait eu lieu " de façon habituelle" (Code S.S. art. L496).

Abstraction faite des éléments médicaux précités, l'exposition habituelle constitue un élément de présomption qui tient à l'essence même de la conception de la maladie comme résultat de la répétition de l'action de l'agent nocif.

\section{ETAT DE LA JURISPRUDENCE ANTÉRIEURE RELATIVE AUX MALADIES DU TABLEAU N*6}

Quoique le nombre de maladies retenues au titre du tableau $n^{\circ} 6$ soit relativement faible, de l'ordre de 17,8 par an pour 4271 maladies professionnelles, en moyenne sur 16 ans, la jurisprudence suscitée par l'application de ce tableau est relativement abondante et marquée par plusieurs arrêts de la Chambre sociale de la Cour de cassation.

Parmi les questions qui ont été soulevées devant les tribunaux, nous retiendrons celle de la nature de la présomption d'origine et d'une articulation entre 
le régime des maladies professionnelles et la réglementation de radioprotection qui se sont trouvées posées par l'application de la notion d'exposition habituelle.

L'arrêt de la Chambre sociale du 10 février 1966 (Dame Majoni), qui a suscité une abondante bibliographie, peut être considéré comme le leading case en la matière.

Cet arrêt est le premier à se prononcer sur la force de la présomption de l'art. L496, alors qu'en matière d'accident du travail, il était déjà admis que la présomption d'imputabilité est une présomption simple.

La preuve contraire est admise, mais il est clair que la volonté du législateur de conforter la position du travailleur serait méconnue si cette preuve était admise trop largement. Le doute, si léger soit-il, sur l'effet du travail sur la lésion ou l'affection bénéficie au travailleur demandeur et non, comme ce serait le cas en responsabilité civile, au défendeur.

En conséquence, la Cour a jugé que le CEA avait la possibilité de rapporter la preuve que la maladie

$1^{\circ}$ était due à une cause autre que la radioactivité professionnelle ;

$2^{\circ}$ que "sans aucun doute possible le risque professionnel n'a eu aucune influence sur son origine ou son évolution".

Les lecteurs de cette revue mesurent aisément la difficulté, le caractère quasi-diabolique, d'une telle preuve en notre domaine.

Un autre motif de l'arrêt doit aussi être retenu. La Cour suprême a approuvé la Cour d'appel qui avait estimé que " même étant admis qu'aient été respectées les règles édictées pour la protection du personnel ", la Dame Majoni avait cependant été exposée de façon habituelle à l'action des rayonnements ionisants, " aucun degré d'intensité ou de dose dangereuse, pour l'action de l'agent nocif, n'ayant été prévu par le tableau n० 6 ".

La Cour pose donc que les réglementations concernant la protection des travailleurs (décret du 15 mars 1967, décret du 28 avril 1975 essentiellement) et la réparation des maladies professionnelles sont indépendantes et séparées en quelque sorte par une cloison étanche. A l'époque, beaucoup de " radioprotecteurs" en furent scandalisés, peut-être par méconnaissance de la fonction régulatrice de la Cour qui consiste à casser les décisions des juridictions inférieures qui lui sont déférées par pourvoi pour violation de la loi soit dans la forme soit dans le fond. Et, effectivement, le tableau $n^{\circ} 6$ actuel ne comporte aucune articulation avec la réglementation de radioprotection.

On objectera, certes, que la jurisprudence de la Cour de cassation s'est souvent montrée novatrice. En l'espèce, la Cour paraît avoir suivi l'Avocat général qui, dans ses conclusions, estimait que l'accueil de "la notion de seuil de nocivité sonnerait le glas du régime juridique actuel des maladies professionnelles", car il conduirait les tribunaux à arbitrer des conflits purement scientifiques entre experts, ce que le législateur, par le régime de présomption, avait voulu éviter.

Les experts, de leur côté, avaient déclaré que la notion de dose maximale admissible " ne tient pas compte des rares fragilités individuelles". 
L'arrêt Majoni et d'autres arrêts de la Chambre sociale ont précisé quelque peu la notion d'exposition habituelle. Celle-ci devant être d'une " certaine régularité et d'une certaine durée " laisse, en effet, place à une marge d'appréciation, en principe de la compétence du juge du fond. Des expositions espacées (par exemple trois en cinq ans pour un total de 75 millirems) ne sont évidemment pas suffisamment régulières (Soc. 18 juin 1970 Saltel). Par contre, dans les autres cas cités ci-après, où les travailleurs avaient été exposés chaque jour ouvrable pendant plusieurs mois, la régularité était acquise.

En fait, c'est surtout sur la durée que la jurisprudence s'est montrée très large en se contentant parfois d'exposition journalière de quelques minutes (Soc. 24 novembre 1976, Vve Georges et aussi arrêt Majoni).

II convient aussi de noter que la dosimétrie individuelle réglementaire constitue un indice qui, rapproché de - ou pour les travailleurs non DATR*, suppléé par- l'examen des conditions concrètes de travail, est pris en considération pour établir cet élément de la présomption.

Ainsi, une documentaliste non-DATR a-t-elle été considérée comme ayant satisfait à la condition habituelle parce que les conditions matérielles de son travail n'assurait pas son isolement contre l'action des rayonnements ionisants (Soc. 2 mars 1978, Dame Belle).

\section{APPORT DE L'ARRET DE LA COUR D'APPEL DE PARIS DU 25 FÉVRIER 1982}

Rappelons les faits de la cause : un travailleur dans un laboratoire de biologie médicale déclare, avec certificats médicaux à l'appui, une radiodermite.

La Commission de recours gracieux de la Sécurité sociale lui reconnut le caractère professionnel. L'employeur contesta devant la Commission de première instance du contentieux le diagnostic, l'exposition habituelle et l'observation des délais, bref se plaça sur le terrain même des éléments de la présomption. Ce n'est qu'à titre subsidiaire qu'il porta son recours sur le terrain de la preuve contraire en plaidant que l'affection aurait été contractée lors d'études suivies par l'assuré. La Commission de première instance, ordonna une expertise médicale judiciaire sur pièce, puis au vu du rapport des experts, déclara fondé le recours de l'employeur.

En appel interjeté par la Caisse devant la Cour de Paris, celle-ci confirma cette décision.

La Cour a relevé que les experts avaient noté des contradictions entre les documents médicaux et s'étaient fait communiquer par la CIREA ${ }^{\star \star}$ les activités des sources fournies au laboratoire, d'où résultait, selon ces experts, que "l'activité des substances radioactives et les conditions dans lesquelles l'assuré les manipulait étaient totalement incompatibles avec une irradiation susceptible de provoquer une radiodermite aussi bien aiguë que chronique, même dans l'hypothèse où il y aurait eu faute intentionnelle ou négligence de la part de rassuré".

* DATR : directement affecté à des travaux sous rayonnements ionisants.

** CIREA : Commission interministérielle des radioéléments artificiels. 
Mais le considérant intéressant nous paraît être le suivant. La Cour reconnaît que le tableau $n^{\circ} 6$, comme la plupart des autres tableaux, ne prévoit pas de seuil d'exposition. Mais il va de soi, dit la Cour, que l'exposition pour être habituelle " doit nécessairement présenter des conditions minimales de fréquence, de durée et d'intensité " et qu'il appartient aux médecins experts de déterminer dans chaque cas, sur la base des données scientifiques ou expérimentales, si la nature des produits et les conditions d'exposition ont été suffisantes pour pouvoir provoquer la maladie.

L'innovation de cet arrêt, par rapport à ceux que nous avons cités plus haut, réside, selon nous, dans cette exigence d'une intensité suffisante et non plus seulement d'une certaine fréquence et d'une certaine durée de l'exposition au risque, pour que celle-ci puisse être considérée comme l'exposition habituelle, élément constitutif de la présomption d'origine.

A cet égard, cet arrêt prend acte de l'évolution survenue depuis 1966. II n'est, en effet, plus possible d'affirmer aujourd'hui, comme l'avait fait l'avocat général dans l'affaire Majoni, que "la notion de seuil de dangerosité" était "étrangère à la matière des maladies professionnelles" alors que plusieurs tableaux ( $n$ os $28,58,64$ ) comportent des seuils d'action de l'agent nocif. La notion d'intensité de l'action des rayonnements comme facteur de l'exposition habituelle apparaît être, à la lecture de l'arrêt, un substitut d'un seuil nonexpressément formulé. L'avantage de la formule réside dans sa souplesse mais corrélativement, elle exige le recours systématique aux experts, ce qui peut apparaître à certains comme une déviation par rapport à l'esprit du système de liste, à son automatisme simplificateur, et rapprochant quelque peu la pratique française du système mixte adopté par certains pays voisins et préconisé par la Commission des Communautés européennes.

\section{CONCLUSION}

II ne conviendrait pas, toutefois, de généraliser imprudemment la solution adoptée par la Cour de Paris.

D'une part, l'arrêt n'a pas fait l'objet d'un pourvoi en cassation et il serait imprudent de présumer que la Cour Suprême infléchisse sa jurisprudence qui, jusqu'à présent (cf. $\S 1$ ci-dessus) n'isolait pas l'intensité (établie par dosimétrie ou autrement) de la fréquence et de la durée d'exposition, lorsqu'elle sera saisie d'un cas analogue.

Sur la preuve contraire, du fait que le débat est resté au stade antérieur de la discussion des éléments de la présomption, l'arrêt s'est borné à rappeler la possibilité d'apporter cette preuve, une fois tous les éléments de la présomption constitués, et il serait également imprudent d'en conclure que la Cour de Paris, et a fortiori la Cour de cassation, assoupliront, à l'avenir, les exigences posées par l'arrêt Dame Majoni.

D'autre part, l'affection alléguée, une radiodermite, est une affection nonstochastique pour laquelle les experts avaient reconnu que l'existence d'un seuil était scientifiquement démontrée.

Pour ce type d'effets, la solution dégagée par la Cour de Paris mériterait d'être reprise à l'occasion, car elle présente un avantage de souplesse, que n'offre pas la solution qui consisterait à insérer des seuils dans le tableau $n^{\circ} 6$, et 
bien qu'elle entraîne une expertise qui n'est pas dans l'esprit de cette législation. Par contre, une application aux effets stochastiques apparaît bien problématique.

Certes, du fait que la jurisprudence (cf. § II supra) pose que les réglementations de radioprotection et de réparation des maladies professionnelles sont distinctes, l'argument selon lequel l'exigence d'une certaine intensité dans l'exposition habituelle serait contraire à l'hypothèse de relation dose-effet sans seuil de la radioprotection serait non concluant. Mais il faudrait que les experts conviennent de seuils pratiques d'effets pour les maladies de ce type, ce qui parait constituer actuellement une proposition hérétique.

L'augmentation des effectifs exposés aux rayonnements ionisants entraînera à terme celle d'affections aussi peu spécifiques que le cancer du poumon parmi les maladies du tableau $n^{\circ} 6$, ce qui pourrait poser des problèmes psychologiques et financiers. La recherche, dès maintenant, d'une solution raisonnable s'impose d'autant plus qu'en dehors du régime des maladies professionnelles, sur le terrain du droit spécial de responsabilité civile nucléaire, la révision prochaine de la Convention de Paris et de la convention Complémentaire de Bruxelles devrait susciter un réexamen de la loi du 30 octobre 1968 qui prévoyait, dans deux hypothèses, que des décrets (non pris) s'inspiraient du système de présomption usité en matière de maladies professionnelles pour résoudre certains problèmes posés par ces Conventions.

\section{BIBLIOGRAPHIE}

Code de la Sécurité Sociale, Livre IV - Titre VI et Annexe IV (Décret n 46.2959 du 31 décembre 1946 modifié) titre VII et tableaux annexés. Petit Code Dalloz, 1980, 118-121. p. 751, p. 758 .

DESPRAIRIES. Problème médico-légaux posés par les radiodermites professionnelles. Thèse, Médecine, Paris, 1972.

TOUTEE et de NERCY. A propos d'un cas de maladie professionnelle attribuée aux rayonnements ionisants. Droit social, 1967, n 1, p. 51.

Cour de Cassation, Chambre Sociale, 10 février 1966, CEA c/Caisse Primaire SSRP et Dame Majoni et Cour d'Appel de Paris, $18^{\circ} \mathrm{Ch} ., 4$ décembre 1965 , mêmes parties, In Dalloz 1966, 305, (Conclusions MELLOTEE) et Juris Classeur Périodique, (JCP), 1967, II, 14923 (avec note J. HEBERT).

Cour d'Appel de Paris, 12 mars 1969, mêmes parties, note J. VERGNE. JCP 1969, II, 16089.

Cour d'Appel de Paris, 8 février 1969, Caisse SSRP c/CEA (Saltel). JCP 1969, II, 16089. note J. VERGNE.

Cour de Cassation, Chambre Sociale, 24 novembre 1976, CEA c/Dame George. Bulletin des Arrêts, Cour de Cassation V, n 682, p. 505.

Cour de Cassation, Chambre Sociale, 2 mars 1978, IN2 P3 (Dame Belle). JCP 1978, IV, 139. 